\title{
Note: Vectorial-magneto optical Kerr effect technique combined with variable temperature and full angular range all in a single setup
}

\author{
Jose Luis F. Cuñado, ${ }^{1,2, a)}$ Javier Pedrosa, ${ }^{2}$ Fernando Ajejas, ${ }^{1}$ Alberto Bollero, ${ }^{2}$ \\ Paolo Perna, ${ }^{2}$ Francisco J. Teran, ${ }^{2}$ Rodolfo Miranda,,${ }^{1,2,3}$ and Julio Camarero ${ }^{1,2,3}$ \\ ${ }^{1}$ Departamento de Física de la Materia Condensada and Instituto "Nicolás Cabrera," Universidad Autónoma \\ de Madrid, 28049 Madrid, Spain \\ ${ }^{2}$ Instituto Madrileño de Estudios Avanzados en Nanociencia IMDEA-Nanociencia, Campus Universidad \\ Autónoma de Madrid, 28049 Madrid, Spain \\ ${ }^{3}$ Condensed Matter Physics Center (IFIMAC), Universidad Autónoma de Madrid, 28049 Madrid, Spain
}

(Received 23 February 2015; accepted 19 April 2015; published online 28 April 2015)

\begin{abstract}
Here, we report on a versatile full angular resolved/broad temperature range/vectorial magneto optical Kerr effect (MOKE) magnetometer, named TRISTAN. Its versatility relies on its capacity to probe temperature and angular dependencies of magnetization reversal processes without the need to do any intervention on the apparatus during measurements. The setup is a combination of a vectorial MOKE bench and a cryostat with optical access. The cryostat has a motorized rotatable sample holder with azimuthal correction. It allows for simultaneous and quantitative acquisition of the two in-plane magnetization components during the hysteresis loop at different temperatures from $4 \mathrm{~K}$ up to $500 \mathrm{~K}$ and in the whole angular range, without neither changing magnet orientation nor opening the cryostat. Measurements performed in a model system with competing collinear biaxial and uniaxial contributions are presented to illustrate its capabilities. (C) 2015 AIP Publishing LLC. [http://dx.doi.org/10.1063/1.4919411]
\end{abstract}

Magneto-optical Kerr effect (MOKE) is widely applied in nanomagnetism research, ${ }^{1-4}$ because of its high sensitivity (down to nanometer thickness), vanishing substrate effects (limited penetration depth), immunity to external fields (photon-in/photon-out approach), and relatively simple implementation. Different Kerr geometries can be used to obtain the different components of magnetization with respect to the applied external field. This normally requires tedious physical changing of optical components, magnet, and/or sample orientation with respect to the optical plane to complete a single vectorial measurement. ${ }^{5-7}$ We have recently presented a vectorial MOKE (v-MOKE) setup which provides simultaneously and quantitatively the two in-plane magnetization components during the hysteresis loop for a given orientation, allowing full angular studies. ${ }^{8}$ It is able to perform automated angular-dependent magnetic measurements of different magnetic nanostructures at room temperature (RT),${ }^{9-15}$ without the need to change the Kerr geometry. When control over temperature is also required, a cryostat must be used. Physical changes, including reflection plane fixing, would then become very difficult, specially when studying magnetic symmetries where angular dependent measurements are needed, and the process must be repeated for each angle in the whole angular range and for each selected temperature.

In this note, we present a variable-temperature vectorialKerr magnetometer, named TRISTAN, that solves these inconveniences. The setup allows full angular range rotation of the sample under controlled temperature, from liquid Helium up to $500 \mathrm{~K}$, and the simultaneous acquisition of the two in-plane magnetization components, i.e., parallel $M_{\|}$and

a)Electronic mail: joseluis.cunnado@uam.es perpendicular $M_{\perp}$ to the external magnetic field $\mu_{0} H$, for any given temperature $T$ and sample orientation $\alpha_{H}$. The measurement process can be carried out in a continuous fully automated way without the need for optical realignment or breaking vacuum. Automation of the process allows to program experiments with different temperature ramps, measurement intervals, field cooling options, as well as with different applied field sweep rates and angular ranges.

The general diagram of TRISTAN instrument is shown in Fig. 1. It consists of a vectorial MOKE setup externally mounted in front of a backflow ${ }^{4} \mathrm{He}$ cryostat with optical access (window). The window is at the end of a cylindrical nose that extends some centimeters away from the main cryostat body, around which the external electromagnet is located. The sample is located inside the cryostat just behind this window, supported by the sample holder which is in thermal contact with a cold finger located in the main body of the cryostat. The sample can be set at a certain controlled temperature by the combined action of the helium flow through the cold finger (controlled with an electromechanical valve located in the ${ }^{4} \mathrm{He}$ backflow pump) and the current on a heating filament in thermal contact with it. The sample holder can be in situ rotated by $360^{\circ}$ and it has been implemented with a tilt-adjustable device to keep fixed the reflection plane upon sample rotation. This is adjusted before closing the cryostat, and no re-adjustments are required even after changing temperatures over the whole temperature range. The miniaturization of these parts is very important in order not to increase the magnet's gap required to enclose the cryostat nose, avoiding a dramatic reduction of the external field applied. The MOKE optical bench setup is of the vectorial type, based on twin photodiodes. ${ }^{8}$ Electromagnet driving, temperature control, axis rotation, and signal acquisition are based on specific electronics connected via GPIB to 


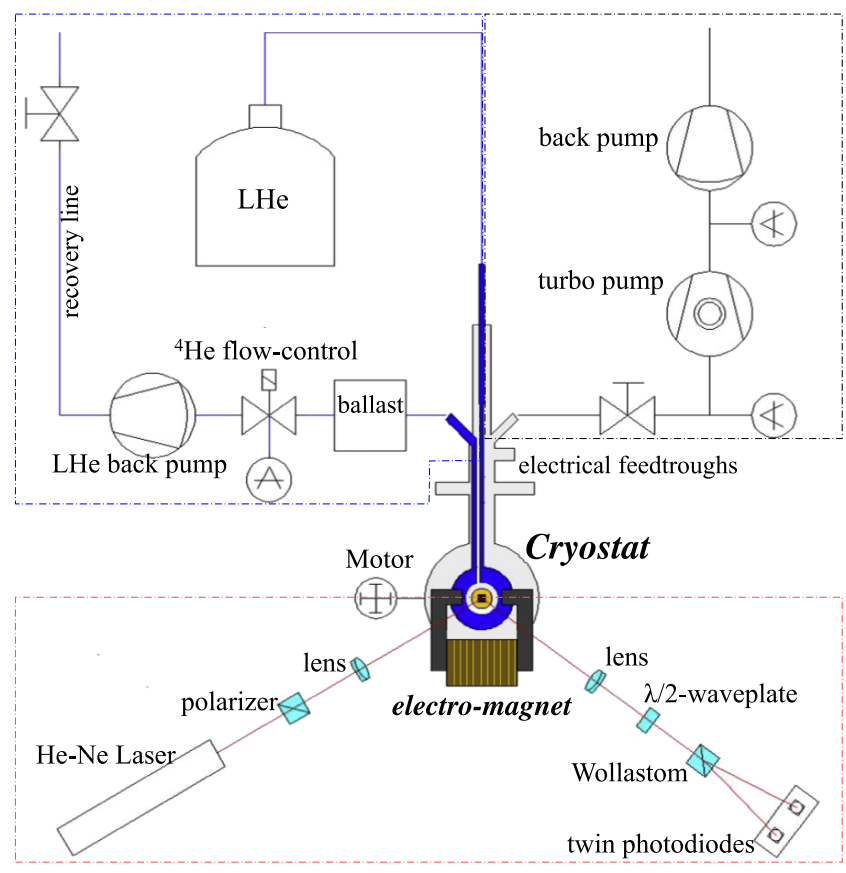

FIG. 1. Schematic layout of TRISTAN main parts, with the cryogenic line (top left quadrant), the vacuum line (top right), the cryostat (middle), and the optical bench with the optical components (bottom).

a computer, with software developed on purpose to automate the control of the instruments and the measurement process.

The cryostat is a single casing (vacuum shield with radiation shield) CryoVac GmbH KONTI Cryostat Micro of the liquid helium backflow type, modified on purpose in the factory according to our specifications, to suit our needs: in situ rotary motion under controlled temperature and vacuum conditions with a $25 \mathrm{~mm}$ diameter optical access window located at the end of a cylindrical extension (the nose). To excite the sample, an external $31 \mathrm{~mm}$ gap electromagnet from evico magnetics $\mathrm{GmbH}$ is located around this extension, without contacting the cryostat or the optical bench. Vacuum better than $10^{-7}$ millibars inside the cryostat is obtained by using a small turbo-molecular pump with membrane backing pump.

The cryogenic liquid is pumped using a backflow rotary vane pump with an electromagnetic valve to control the flow. Vacuum and liquid He hoses have been provided with ballast to avoid vibrations coming from the pumps. Electrical feedthroughs for temperature sensors, heater, and additional contacts are also provided in the cryostat. The sample holder is attached to a rotary axis that can be actuated to the desired angle. Four flexible oxygen-free $\mathrm{Cu}$ braids put the sample holder in thermal contact with the cold finger, acting efficiently as heat exchanger and allowing the required sample rotation. Two temperature sensors are located in the heat exchanger and in the sample holder neck. Fast and efficient stabilization of the temperature in the sample is obtained by the combined action of the heater filament located in the heat exchanger and the cryogenic flow, controlled by a PID (proportional-integralderivative) electronic device.

Some additional modifications have been developed in our laboratory to improve the capabilities of the cryostat. We have designed and built a $15 \mathrm{~mm}$ diameter eucentric stage, to replace the original sample holder, with a set of screws and springs to keep fixed the reflection plane upon sample rotation and without breaking thermal contact. A third temperature sensor is located in the sample holder just at the position of the sample clamp. An additional structure to support the motor assembly in the rotary knob at the rear part of the cryostat has been designed and built. The cryostat is fixed with a base placed in the middle of one side of the optical bench and with the nose axis pointing to the center of the bench. The base includes adjustable micrometric XYZ translation stages. The optical bench is provided with passive pneumatic dumpers to minimize building vibrations (in the range of $0.1-10 \mathrm{~Hz}$ ).

In order to illustrate the capabilities of TRISTAN, we present measurements performed in a model Fe(001) thin film with competing collinear four-fold (biaxial) and two-fold (uniaxial) anisotropy contributions. The Fe film was grown at oblique incidence on a $\mathrm{MgO}(100)$ single crystal in order to induce magnetic symmetry breaking effects. The cubic crystal structure of the $\mathrm{Fe}(001)$ film promotes an in-plane biaxial (four-fold) magnetic anisotropy with magnetic easy axes at [100] and [010] directions. Oblique deposition gives rise to an additional in-plane (two-fold) uniaxial magnetic anisotropy with the anisotropy axis oriented perpendicular to the deposition direction. ${ }^{16}$

In general, the competition between four-fold and twofold anisotropy contributions depends on intrinsic parameters, such as angle of deposition, ${ }^{16}$ substrate step density, ${ }^{17}$ and ferromagnetic thicknesses ${ }^{18}$ and/or extrinsic ones, such as temperature. Different temperature dependences would result in a spin-reorientation transition. In our case, at RT, the expected four-fold (biaxial) magnetic symmetry of the $\mathrm{Fe}(001)$ film is broken by an additional two-fold (uniaxial) contribution with the easy axis aligned with one of the easy axes of the biaxial one, referred as e.a.I. The effect of this uniaxial contribution vanishes as temperature decreases. Detailed discussion of thermal effects on magnetic symmetries will be reported

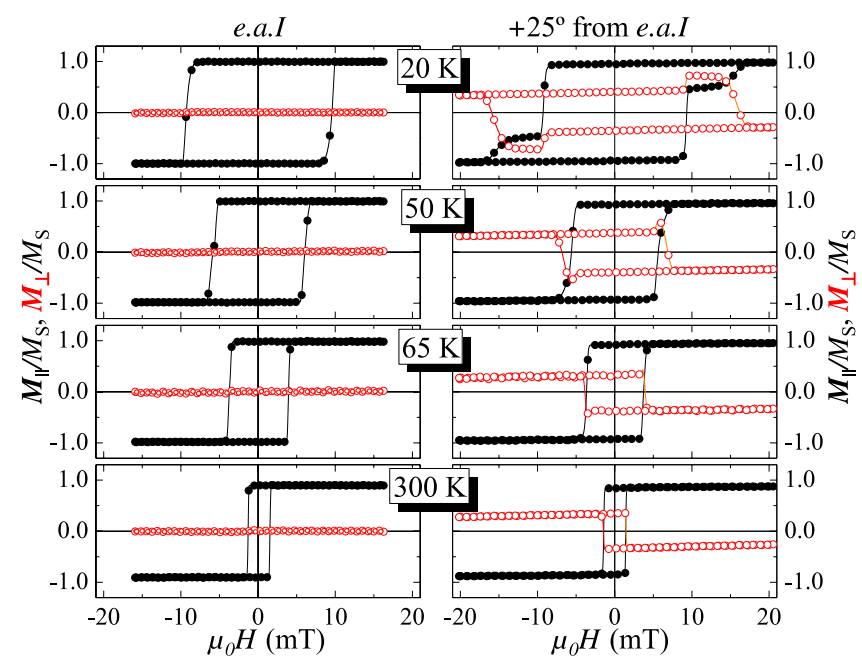

FIG. 2. Representative temperature dependent in-plane resolved hysteresis loops at two selected angles, e.a.I. (left graphs) and $-25^{\circ}$ off e.a.I (right graphs) of a $\mathrm{Fe}(001)$ film with competing collinear biaxial and growthinduced uniaxial anisotropies. $M_{\|}(H, T)$ and $M_{\perp}(H, T)$ loops are represented by filled and empty symbols, respectively. Notice the change of reversal pathway from above $60 \mathrm{~K}$ at $\alpha_{H}=-25^{\circ}$. 
elsewhere. Here, we show relevant experimental data in order to illustrate the capabilities of the setup.

Representative temperature-dependent in-plane vectorial resolved hysteresis loops acquired at two different angles are shown in Fig. 2. $\alpha_{H}=0^{\circ}$ refers to the in-plane [100] crystal direction of the $\mathrm{Fe}(001)$ film aligned parallel to the field direction. The angles have been selected in order to show two very different temperature evolutions in the same magnetic system. For $\alpha_{H}=0^{\circ}$, i.e., at e.a.I, the shape of the hysteresis loop does not vary with $\mathrm{T}$, whereas its coercivity $H_{\mathrm{C}}$ decreases as temperature increases (left graphs of Fig. 2). This behaviour is the expected one for thermally activated processes. For all temperatures, one irreversible transition with a negligible perpendicular component indicates that the reversal takes place via nucleation of antiparallel domains aligned with the anisotropy axis and fast propagation of $180^{\circ}$ domain walls (DWs). Therefore, thermally assisted reversal would explain the temperature evolution without introducing any symmetrybreaking effect.

A very different scenario is found at $\alpha_{H}=+25^{\circ}$ (right graphs of Fig. 2), where different magnetization reversal pathways are found. In particular, both parallel and perpendicular components show that the reversal takes place via two consecutive irreversible transitions and one irreversible transition below and above $60 \mathrm{~K}$, respectively. The former is characteristic of well-defined biaxial (four-fold) magnetic anisotropy systems, where each irreversible transition corresponds to nucleation and further $90^{\circ} \mathrm{DW}$ propagation, as expected for a $\mathrm{Fe}(001)$ film. In the two temperature ranges, the reversal fields decrease as the temperature increases, i.e., thermally activated processes. The magnetization reverses via nucleation and further $90^{\circ}\left(180^{\circ}\right) \mathrm{DW}$ propagation below (above) the critical temperature. Therefore, the low temperature biaxial magnetic anisotropy is dominated by another anisotropy contribution at high temperature. In fact, the film was grown at oblique incidence on purpose, in order to induce this additional uniaxial magnetic anisotropy. The full understanding of this temperature-dependent symmetry-breaking effect hence requires both temperature and angle dependent measurements.

In summary, we have introduced a versatile automated variable-temperature vectorial-MOKE setup, named TRISTAN, with working temperature range from $4 \mathrm{~K}$ to $500 \mathrm{~K}$ and full angular range. TRISTAN provides simultaneous and quantitative vectorial capability for any given angle and temperature and allows to carry out detailed angular and temperature experiments of magnetic nanostructures under controlled conditions (temperature, magnetic history, field cooling, dynamics, and signal switching). TRISTAN allows extracting relevant magnetic parameters from the vectorial-resolved hysteresis curves acquired at different temperatures and angles. More remarkably the set-up provides direct views on magnetization reversal processes and magnetic transitions, enabling us to unravel magnetic symmetry-breaking effects of magnetic nanostructures.

We thank the UAM research support services, SEGAINVEX, for the detectors and power supplies development and gratefully acknowledge to the technical staff of the UAM Condensed Matter Department, specially to Santiago Márquez, Andrés Buendía, and José Luis Romera. We also thank Hermann Suderow and Isabel Guillamón from LBTUAM for their support in low temperature aspects. P.P. acknowledges financial support from MINECO through Contract No. JCI-201109602. F.J.T. acknowledges financial support from Ramon y Cajal program (RYC-2011-09617). This work has been supported by MINECO through Project Nos. MAT2011-25598 and MAT2012-39308, by the Comunidad de Madrid through Project No. S2013/MIT-2850 NANOFRONTMAG-CM and by EU-FP7 through NANOPYME Project (No. 310516).

${ }^{1}$ J. Kerr, Philos. Mag. Ser. 5 3, 321 (1877).

${ }^{2}$ E. R. Moog and S. D. Bader, Superlattices Microstruct. 1, 543 (1985).

${ }^{3}$ Z. Q. Qiu and S. D. Bader, J. Magn. Magn. Mater. 200, 664 (1999); Rev. Sci. Instrum. 71, 1243 (2000).

${ }^{4}$ A. Hubert and R. Schäfer, Magnetic Domains (Springer-Verlag, Berlin).

${ }^{5}$ J. M. Florczak and E. Dan Dahlberg, J. Appl. Phys. 67, 7520 (1990).

${ }^{6}$ C. Daboo, J. A. C. Bland, R. J. Hicken, A. J. R. Ives, M. J. Baird, and M. J. Walker, Phys. Rev. B 47, 11852 (1993).

${ }^{7}$ T. Kuschel, H. Bardenhagen, H. Wilkens, R. Schubert, J. Hamrle, J. Pistora, and J. Wollschläger, J. Phys. D: Appl. Phys. 44, 265003 (2011).

${ }^{8}$ E. Jiménez, N. Mikuszeit, J. L. F. Cuñado, P. Perna, J. Pedrosa, D. Maccariello, C. Rodrigo, M. A. Niño, A. Bollero, J. Camarero, and R. Miranda, Rev. Sci. Instrum. 85, 053904 (2014).

${ }^{9}$ J. Camarero, J. Sort, A. Hoffmann, J. M. García-Martín, B. Dieny, R. Miranda, and J. Nogués, Phys. Rev. Lett. 95, 057204 (2005).

${ }^{10}$ T. Bobek, N. Mikuszeit, J. Camarero, S. Kyrsta, L. Yang, M. A. Niño, C. Hofer, L. Gridneva, D. Arvanitis, R. Miranda, J. J. de Miguel, C. Teichert, and H. Kurz, Adv. Mater. 19, 4375 (2007).

${ }^{11}$ P. Prieto, K. R. Pirota, J. M. Sanz, E. Jiménez, F. Maccherozzi, and F. G. Panaccione, Appl. Phys. Lett. 90, 032505 (2007).

${ }^{12}$ D. Ecija, E. Jiménez, N. Mikuszeit, N. Sacristán, J. Camarero, J. M. Gallego, J. Vogel, and R. Miranda, Phys. Rev. B 77, 024426 (2008); J. Magn. Magn. Mater. 316, 321 (2007).

${ }^{13}$ E. Jiménez, J. Camarero, J. Sort, J. Nogués, A. Hoffmann, N. Mikuszeit, J. M. García-Martín, B. Dieny, and R. Miranda, Phys. Rev. B 80, 014415 (2009); Appl. Phys. Lett. 95, 122508 (2009).

${ }^{14}$ P. Perna, C. Rodrigo, E. Jiménez, N. Mikuszeit, F. J. Teran, L. Méchin, J. Camarero, and R. Miranda, J. Appl. Phys. 110, 089903 (2011).

${ }^{15}$ P. Perna, C. Rodrigo, M. Muñoz, J. L. Prieto, A. Bollero, D. Maccariello, J. L. F. Cuñado, M. Romera, J. Akerman, E. Jiménez, N. Mikuszeit, V. Cros, J. Camarero, and R. Miranda, Phys. Rev. B 86, 024421 (2012); Appl. Phys. Lett. 104, 202407 (2014).

${ }^{16}$ Y. Park, E. E. Fullerton, and S. D. Bader, Appl. Phys. Lett. 66, 2140 (1995).

${ }^{17}$ R. K. Kawakami, E. J. Escorcia-Aparicio, and Z. Q. Qiu, Phys. Rev. Lett. 77, 2570-2573 (1996).

${ }^{18}$ F. Bisio, R. Moroni, F. Buatier de Mongeot, M. Canepa, and L. Mattera, Phys. Rev. Lett. 96, 057204 (2006). 